\title{
The Unified Approach to Spectral Analysis
}

\author{
R. Weder
}

Princeton University, Princeton, New Jersey 08540, USA

\begin{abstract}
We develop a new, unified, method to construct a closed (selfadjoint in $\mathscr{L}^{2}$ ) extension of a partial differential operator in all the spaces $\mathscr{L}^{p}\left(\mathbb{R}^{n}\right) 1 \leqq p \leqq \infty$. Our method is not only an unified approach but it is also very efficient. We obtain very weak conditions on the potentials.
\end{abstract}

\section{Introduction}

In this paper we develop a new method to construct a closed extension (selfadjoint in the case $\mathscr{L}^{2}$ ) of a partial differential operator in $\mathscr{L}^{p}\left(R^{n}\right), 1 \leqq p \leqq \infty$, and to study its spectral properties.

Let $P_{0}$ be a constant coefficients partial differential operator and let $Q=\sum_{i=1}^{M} q_{i} Q_{i}$ be a lower order variable coefficients partial differential operator. The basic object of our method is the formal series expansion for the resolvent of the perturbed operator $P_{0}+Q$ :

$$
R(z)=\sum_{n=0}^{\infty} R_{0}(z)\left(Q R_{0}(z)\right)^{n} .
$$

$R_{0}(z)$ being the resolvent of the unperturbed operator $P_{0}$.

Our strategy is to prove, by an interpolation argument, that the formal series expansion defines a bounded operator in all the $\mathscr{L}^{p}\left(\mathbb{R}^{n}\right)$ spaces, $1 \leqq p \leqq \infty$, denoted $R_{p}(z)$. Then we prove that $R_{p}(z)$ is the resolvent of a closed extension of the perturbed operator $P_{0}+Q$.

Several methods have been proposed to construct a closed extension (selfadjoint in $\mathscr{L}^{2}$ ) of a partial differential operator in $\mathscr{L}^{p}, 1 \leqq p \leqq \infty$. In $\mathscr{L}^{p}, 1 \leqq p<\infty$, if the potentials $q_{i}(x)$ are locally in $\mathscr{L}^{p}$, the closed extension is defined as the operator sum of $P_{0}$ and $Q$. For a general treatment of this method see [3] were references to original contributions are given.

The method of quadratic forms extensions in $\mathscr{L}^{2}$ has been developed by [2] (and the references to original contributions quoted there) [5], [6], [7], and [8]. In $\mathscr{L}^{p}, 1<p<\infty$, the method of $S$-extensions has been created by M. Schechter [3]. 
A theory to deal with the case $\mathscr{L}^{\infty}$ has been created in [9] and [1].

The fact that, for technical reasons, different methods are necessary to construct a closed extension in different $\mathscr{L}^{p}$ spaces is unpleasant because the basic object is the partial differential operator not the $\mathscr{L}^{p}$ space in which it is defined.

Our approach has the interesting new feature that it provides an unified method to construct a closed extension, selfadjoint in $\mathscr{L}^{2}$, in all $\mathscr{L}^{p}$ spaces, $1 \leqq p \leqq \infty$.

We give our conditions in terms of the quantity

$$
R_{\alpha}(q)=\sup _{x} \int|q(x-y)| G_{\alpha}(y) d y .
$$

$R_{\alpha}$ is the class functions such that $R_{\alpha}(q)<\infty$.

Where:

$$
G_{\alpha}(x)=F^{-1}\left(\frac{Q_{\alpha}(k)}{i+P_{0}(k)}\right) .
$$

By $F$ we denote the Fourier transform. We assume

A) Assume that $q_{\alpha}(x) \in R_{\alpha}$ and that $\lim _{R \rightarrow \infty} \int_{|y|>R}\left|q_{\alpha}(y)\right| G_{\alpha}(y-x) d y=0$ uniformly in $x$. We are interested in formally symmetric operators. ${ }^{1}$ This is not a serious restriction since in most of the applications the operators are formally symmetric. Our main Theorem is:

Theorem 1.1. Let $P_{0}$ be elliptic of degree $m$, let $Q_{\alpha}$ be of degree $l_{\alpha}<m$, and let $P=P_{0}+\sum_{\alpha=1}^{M} q_{\alpha} Q_{\alpha}$ be formally symmetric. Assume that $(A)$ is satisfied. Then $P$ defined on $D(P)=\left\{\phi \in C_{0}^{\infty} \mid P \phi \in \mathscr{L}^{p}\right\}$ has a closed extension, denoted $H_{p}$, in $\mathscr{L}^{p}$ for $1 \leqq p \leqq \infty . H_{p}$ is densely defined for $1 \leqq p<\infty$, and $H_{p}^{*}=H_{p^{\prime}}, \frac{1}{p}+\frac{1}{p^{\prime}}=1$, $1 \leqq p<\infty$. In particular $\mathrm{H}_{2}$ is selfadjoint. Moreover the essential spectrum of $H_{p}$ is given by $\sigma_{e}\left(H_{p}\right)=\left\{P_{0}(k) \mid k \in \mathbb{R}^{n}\right\}, 1 \leqq p \leqq \infty$. If $\operatorname{dist}\left(z, \sigma_{e}\left(H_{p}\right)\right.$ is large enough the formal series expansion for the resolvent is convergent in norm, i.e.

$$
\left(z-H_{p}\right)^{-1}=\sum_{n=0}^{\infty} R_{0}(z)\left(Q R_{0}(z)^{n}\right.
$$

Theorem I.1 tells us that if (A) is satisfied $P$ has a closed extension, with the same essential spectrum, in all the $\mathscr{L}^{p}$ spaces, $1 \leqq p \leqq \infty$. This result is to be expected since the operator is the basic object, not the $\mathscr{L}^{p}$ space, but to our knowledge this is the first time that such a statement is proved.

Our method is not only a unified approach, but it is also efficient. We obtain very weak conditions in the potential $q_{\alpha}$. Condition $(\mathrm{A})$ is much weaker than the conditions obtained by the previously known methods.

In Theorem II.9 we prove that our method gives in $\mathscr{L}^{2}$ the same result that the method of quadratic forms extensions, i.e. that $H_{2}=H, H$ being the self-

1 My method extends trivially to the case when $P_{0}+Q$ is not formally symmetric 
adjoint extension obtained by quadratic forms methods.

In Theorem II.10 we prove that the spectrum of $H_{p}, 1 \leqq p \leqq \infty$, is real.

Finally let us mention that we proved the following Theorem in the interpolation of compacity ${ }^{2}$ :

Theorem I.2. Let $H$ be a compact operator from $\mathscr{L}^{p_{1}}\left(\Omega_{1}\right)$ to $\mathscr{L}^{q_{1}}\left(\Omega_{2}\right)$ and bounded from $\mathscr{L}^{p_{2}}\left(\Omega_{1}\right)$ to $\mathscr{L}^{q_{2}}\left(\Omega_{2}\right), 1 \leqq p_{1}, p_{2}, q_{1}, q_{2} \leqq \infty$. Where $\Omega_{1}$ and $\Omega_{2}$ are (possibly unbounded) subsets of $\mathbb{R}^{n}$. Then if $q_{1}<\infty H$ is compact from $\mathscr{L}^{p_{t}}\left(\Omega_{1}\right)$ to $\mathscr{L}^{q_{t}}\left(\Omega_{2}\right)$, $0<t<1$, where

$$
\frac{1}{p_{t}}=\frac{t}{p_{1}}+\frac{(1-t)}{p_{2}}, \frac{1}{q_{t}}=\frac{t}{q_{1}}+\frac{(1-t)}{q_{2}} .
$$

This theorem is interesting in its own right. In a forthcoming paper [10] we extend the results of this paper to a large class of partial differential operators introduced in [3]. The method developed in this paper has the advantage over quadratic forms that the unperturbed operator is not required to be bounded below (or sectorial). Of course elliptic operators are, up to a sign, bounded below, but my method extends immediately to situations where the unperturbed operator is not bounded below, for example to systems of equations.

\section{The Proofs}

Let $P_{0}$ denote a constant coefficients elliptic partial differential operator of degree $m$ :

$$
\begin{aligned}
& P_{0}=\sum_{|\mu| \leqq m} a_{\mu} D_{\mu} \\
& \mu=\left(\mu_{1}, \mu_{2}, \ldots, \mu_{n}\right) ; D_{\mu}=\left(D_{1}^{\mu_{1}}, \ldots, D_{n}^{\mu_{n}}\right) \\
& D_{k}=-i \frac{\partial}{\partial x_{k}}, x=\left(x_{1}, \ldots, x_{n}\right) \in R^{n} . \text { We denote also } \\
& P_{0}(k)=\sum_{|\mu| \leqq m} a_{\mu} k^{\mu} .
\end{aligned}
$$

$P_{0}(k)$ is assumed to be a real valued function on $\mathbb{R}^{n}$.

$\mathrm{P}_{0}(D)$ is defined in the set (denoted $C_{0}^{\infty}$ ) of infinitely differentiable functions of compact support.

For $\mathscr{L}^{p}, 1 \leqq p<\infty, \mathscr{L}^{p}$ is densely defined and closable. We denote its closure by $P_{0, p} . P_{0}$ is closable in $\mathscr{L}^{\infty}$ but it is not densely defined. The domain of its closure is too small. For this reason we consider the weak extension in $\mathscr{L}^{\infty}$ instead of the closure, i.e. we define

$$
P_{0, \infty}=P_{0,1}^{*} \text {. }
$$

$P_{0, \infty}$ is obviously closed and is an extension of $P_{0}$.

For a linear operator $H$ in a Banach space $B$ we define the resolvent set $\rho(H)$ and the spectrum $\sigma(H)$ in the usual way. We denote by $\sigma_{d}(H)$ the discrete spectrum

2 See Remark II.8. 
of $H$, i.e. the set of isolated eigenvalues of finite multiplicity. Finally:

Definition II.1. The essential spectrum $\sigma_{e}(H)$ of a linear operator $H$ is the complement (in the spectrum) of the discrete spectrum, i.e. $\sigma_{e}(H)=\sigma(H) \backslash \sigma_{d}(H)$. We have:

\section{Lemma II.2.}

$$
\sigma\left(P_{0, p}\right)=\sigma_{e}\left(P_{0, p}\right)=\left\{P_{0}(k) \mid k \in \mathbb{R}^{n}\right\} .1 \leqq p \leqq \infty .
$$

Proof. For $p<\infty$ this is a classical result. The case $p=\infty$ follows as in [9].

We denote by $R_{0}(z)$ the following operator

$$
\left(R_{0}(z) f\right)(x)=\int F^{-1}\left(\frac{1}{z-P_{0}(k)}\right)(x-y) f(y) d y, \text { for } z \in \mathbb{C} \backslash\left\{P_{0}(k) \mid k \in \mathbb{R}^{n}\right\} .
$$

Lemma II.3. $R_{0}(z)$ is a bounded operator on $\mathscr{L}^{p}$, and $R_{0}(z)=\left(z-P_{0, p}\right)^{-1}$, for $1 \leqq p \leqq \infty$.

Proof. Since $\left|D^{\mu} \frac{1}{P_{0}(k)}\right| \leqq \frac{C}{|k|^{\mu+m}},|k| \rightarrow \infty, F^{-1}\left(\frac{1}{z-P_{0}(k)}\right) \in \mathscr{L}^{1}$. Then $R_{0}(z)$ is a bounded operator on $\mathscr{L}^{p}$. The rest of the Lemma follows as in [1].

The perturbation, $Q$, is the following operator

$$
Q=\sum_{i=\alpha}^{M} q_{\alpha}(x) Q_{\alpha} .
$$

The $Q_{\alpha}$ are constant coefficients partial differential operators of degree, $l_{\alpha}$, smaller than $m$. Let $P$ denote the perturbed operator

$$
P=P_{0}+Q \text {. }
$$

We are interested in operators which are formally symmetric, i.e. such that

$$
(P \phi, \psi)=(\phi, P \psi), \phi, \psi \in D(P) .
$$

This is not a serious restriction. In fact in most of the applications the operators are formally symmetric.

The basic object of our method is the formal series expansion for the resolvent of the perturbed operator

$$
R(z)=\sum_{n=0}^{\infty} R_{0}\left(Q R_{0}\right)^{n}=\sum_{n=0}^{\infty}\left(R_{0} Q\right)^{n} R_{0} .
$$

The strategy of our method is to prove that the formal series expansion defines a bounded operator in $\mathscr{L}^{p}, 1 \leqq p \leqq \infty$, denoted $R_{p}(z)$. Then we prove that $R_{p}(z)$ is a resolvent, i.e.

$$
R_{p}(z)=\left(z-H_{p}\right)^{-1} \text {. }
$$

Finally we prove that $H_{p}$ is a closed extension of the perturbed operator $P$ defined on the set of functions $\phi \in C_{0}^{\infty}$ such that $P \phi \in \mathscr{L}^{p}$. We give our conditions in terms 
of the following quantity:

$$
R_{\alpha}(q)=\sup \int|q(x-y)| G_{\alpha}(y) d y .
$$

$R_{\alpha}$ is the class of functions such that $R_{\alpha}(q)<\infty$. Where:

$$
G_{\alpha}(x)=F^{-1}\left(\frac{Q_{\alpha}(k)}{i+P_{0}(k)}\right) .
$$

Since $\left|D^{\mu}\left(Q_{\alpha}(k) / P_{0}(k)\right)\right| \leqq \frac{C}{|k|^{|\mu|+m-l_{\alpha}}},|k| \rightarrow \infty, G_{\alpha} \in \mathscr{L}^{1}$.

We split the proof of Theorem I.1 in several Lemmas.

Lemma II.4. Let $(A)$ be satisfied. If $\operatorname{dist}\left(z,\left\{P_{0}(k) \mid k \in \mathbb{R}^{n}\right\}\right)$ is large enough the series expansion for the resolvent converges in norm to the resolvent of a closed operator $H_{p}$ in $\mathscr{L}^{p}, 1 \leqq p \leqq \infty . H_{p}$ is a closed extension of $P$.

Proof. As in Lemma III.2 of [1] we prove that $Q$ is $P_{0.1}$ compact. Hence $H_{1}=$ $P_{0,1}+Q$ is closed in $D\left(H_{1}\right)=D\left(P_{0,1}\right)$. Moreover $Q R_{0}(z)$ is bounded in $\mathscr{L}^{1}$ and if $\operatorname{dist}\left(z,\left\{P_{0}(k) \mid k \in \mathbb{R}^{n}\right\}\right)$ is large enough $\left\|Q R_{0}(z)\right\|<1$ as operator in $\mathscr{L}^{1}$. Then

$$
R_{1}(z)=R_{0}(z)\left(1-Q R_{0}\right)^{-1}=\sum_{n=0}^{\infty} R_{0}(z)\left(Q R_{0}(z)\right)^{n} .
$$

The series being absolutely convergent. This proves the Lemma in $\mathscr{L}^{1}$. Since $P$ is formally symmetric $R_{0}(z) Q=\left(Q R_{0}(z)\right)^{*}$, i.e. $R_{0}(z) Q$ is a contraction in $\mathscr{L}^{\infty}$. Hence the formal series is absolutely convergent to a bounded operator in $\mathscr{L}^{\infty}$

$$
R_{\infty}(z)=\sum_{n=0}^{\infty}\left(R_{0}(z) Q\right)^{n} R_{0}(z)=\left(1-R_{0} Q\right)^{-1} R_{0} .
$$

$R_{\infty}$ is clearly injective. Moreover $R_{\infty}$ satisfies the resolvent equation since $R_{\infty}(z)=$ $R_{1}^{*}(\bar{z})$ and $R_{1}(z)$ is a resolvent. Then $R_{\infty}$ is the resolvent of a closed operator, $H_{\infty}$, i.e. $R_{\infty}(z)=\left(z-H_{\infty}\right)^{-1}$. Let us prove that $H_{\infty}$ is an extension of $P$ in $\mathscr{L}^{\infty}$. Let $\phi \in C_{0}^{\infty}$ and $P \phi \in \mathscr{L}^{\infty}$. Denote $\psi=(z-P) \phi$. Then $R_{\infty}(z) \psi=\sum_{n=0}^{\infty}\left(R_{0} Q\right)^{n} R_{0} \psi=$ $\sum_{n=0}^{\infty}\left(R_{0} Q\right)^{n} \phi-\sum_{n=1}^{\infty}\left(R_{0} Q\right)^{n} \phi=\phi$. Then $\phi \in D\left(P_{\infty}\right)$ and $P_{\infty} \phi=P \phi$. This proves the

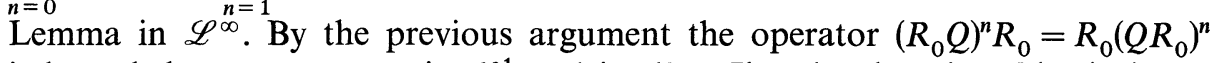
is bounded as an operator in $\mathscr{L}^{1}$ and in $\mathscr{L}^{\infty}$. Then by the Riesz Thorin interpolation theorem is also bounded in $\mathscr{L}^{p}, 1<p<\infty$. For the same reason the formal series expansion converges in norm to a bounded operator, denoted $R_{p}(z)$, in $\mathscr{L}^{p}, 1<p<\infty$.

Since $R_{p}$ satisfies the resolvent equation in $\mathscr{L}^{1}$ and $\mathscr{L}^{\infty}$ it satisfies the resolvent equation in $\mathscr{L}^{p} 1<p<\infty$, by interpolation.

Assume $R_{p}(z) \phi=0$. Then

$$
0=R_{0} Q R_{p} \phi=R_{p} \phi-R_{0} \phi=-R_{0} \phi=0 .
$$

Then $\phi=0$ and $R_{p}$ is injective. 
Then $R_{p}(z)$ is the resolvent of a closed operator $H_{p}$

$$
R_{p}(z)=\left(z-H_{p}\right)^{-1} \text {. }
$$

We prove that $H_{p}$ is an extension of $P$ in $\mathscr{L}^{p} 1<p<\infty$ as we $\operatorname{did}$ for $\mathscr{L}^{\infty}$.

Lemma II.5. The operators $H_{p}$ are densely defined and

$$
H_{p}^{*}=H_{p^{\prime}} \frac{1}{p}+\frac{1}{p^{\prime}}=1 \text { for } 1 \leqq p<\infty .
$$

Proof. Denote $D_{p}=$ Range $R_{p}$ for $1 \leqq p<\infty$. Let $\phi \in \mathscr{L}^{p}$ and let $d=\operatorname{dist}\left(\phi, D_{p}\right)$. Then by the Hahn-Banach Theorem there exists a $\psi \in \mathscr{L}^{p^{\prime}}$ such that $(\psi, \phi)=d$ and $(\psi, \chi)=0$ for all $\chi \in D_{p}$. But if $\chi \in D_{p} \chi=R_{p} \omega$ for some $\omega \in \mathscr{L}^{p}$. Then $(\psi, \chi)=$ $\left(\psi, R_{p}(z) w\right)=\left(R_{p^{\prime}}(\bar{z}) \psi, \omega\right)=0$ for all $\omega \in \mathscr{L}^{p}$. Hence $R_{p^{\prime}}(\bar{z}) \psi=0 \Rightarrow \psi=0$. Then $d=$ dist $\left.\phi, D_{p}\right)=(\psi, \phi)=0$, and $D_{p}$ is dense in $\mathscr{L}^{p}$, for $1 \leqq p<\infty$. Since $R_{p}^{*}(z)=$ $R_{p^{\prime}}(z), 1 \leqq p<\infty$, we have $H_{p}^{*}=\stackrel{p}{H}_{p^{\prime}}$.

We say that a closed operator $\stackrel{p}{H}$ in a Banach space $\mathscr{B}$ is semi-Fredholm [2] if Range $H$ is closed and one of nul $H$ or def $H$ is finite. The index of $H$ is defined by ind $H=\operatorname{nul} H-\operatorname{def} H$, if either nul $H$ or def $H$ is finite.

We denote by $\phi_{H}$ the set of complex numbers $z$ such that $z-H$ is semiFredholm [2].

$\phi_{H}$ is the union of a countable number of connected open sets (components).

Lemma II.6. Let $A$ be a closed operator in a Banach space having the property that in each of the components of $\phi_{A}$ there is at least one point in the resolvent set of $A$. Let $B$ be a closed operator having the same property. Then if there is a $\lambda \in \rho(A) \cap$ $\rho(B)$ such that $(\lambda-A)^{-1}-(\lambda-B)^{-1}$ is compact $\sigma_{e}(A)=\sigma_{e}(B)$.

Proof. Since $A$ has at least one point in the resolvent set in each component of $\phi_{A}$ it can only have discrete spectrum on $\phi_{A}$. Then $\sigma_{e}(A)=\psi_{A}=\mathbb{C} \backslash \phi_{A}$. By the same argument

$$
\sigma_{e}(B)=\psi_{B}=\mathbb{C} \backslash \phi_{B}
$$

But if $(\lambda-A)^{-1}-(\lambda-B)^{-1}$ is compact $\phi_{A}=\phi_{B}$, then it follows that

$$
\sigma_{e}(A)=\sigma_{e}(B)
$$

Lemma II.7. If $A$ is satisfied:

$$
\sigma_{e}\left(H_{p}\right)=\left\{P_{0}(k) \mid k \in \mathbb{R}^{n}\right\} .1 \leqq p \leqq \infty .
$$

Proof. As in Lemma III.2 of [1] we prove that $Q$ is $P_{0,1}$ compact, then

$Q R_{0}$ is compact in $\mathscr{L}^{1}$ and $R_{1}(z)-R_{0}(z)=\sum_{i=1}^{n} R_{0}(z)\left(Q R_{0}(z)^{n}\right.$ is compact in $\mathscr{L}^{1}$. By taking adjoints $R_{\infty}-R_{0}$ is compact in $\mathscr{L}^{\infty}$. Then by Theorem $\mathrm{I}$.2 proved below $R_{p}(z)-R_{0}(z)$ is also compact in $\mathscr{L}^{p}, 1<p<\infty$. Then it follows from Lemma II. 6 that

$$
\sigma_{e}\left(P_{p}\right)=\sigma_{e}\left(P_{0, p}\right)=\left\{P_{0}(k) \mid k \in \mathbb{R}^{n}\right\}
$$


Proof of Theorem I.1. It follows from Lemmas II.4, II.5, and II.7.

Let us prove now Theorem I.2.

Proof of Theorem I.2. Given a subset of $\mathbb{R}^{n}, \Omega$, denote by $\chi_{\Omega}$ the characteristic function of $\Omega$. Let $\Omega_{n}$ be a sequence of bounded sets such that $\Omega_{n} \subset \Omega_{2}$ and $\chi_{\Omega_{n}}(x)$ $\longrightarrow \chi_{\Omega_{2}}$. Let $P_{n}$ be the operator of multiplication by $\chi_{\Omega_{n}}$ in $\mathscr{L}^{q_{t}} . P_{n}$ is a projector. $R \rightarrow \infty$

Moreover $P_{n}$ converges strongly to the identity in $\mathscr{L}^{q_{t}}$.

Since $\Omega_{n}$ is bounded $P_{n} H$ is compact from $\mathscr{L}^{p_{t}}\left(\Omega_{1}\right)$ to $\mathscr{L}^{q_{t}}\left(\Omega_{n}\right)$ by a result of Krasnosel'skii [4]. Since $\stackrel{h}{H}$ is compact from $\mathscr{L}^{p_{1}}\left(\Omega_{1}\right)$ to $\mathscr{L}^{q_{1}}\left(\Omega_{2}\right) P_{n} H$ converges in norm to $H$ as operator from $\mathscr{L}^{p_{1}}$ to $\mathscr{L}^{q_{1}}$. By the Riesz Thorin Theorem $\left\|P_{n} H-H\right\|_{p_{t}, q_{t}} \leqq 2\left\|P_{n} H-H\right\|_{p_{1}, q_{1}}^{1-t}\|H\|_{p_{2}, q_{2}}^{t} \underset{n \rightarrow \infty}{\longrightarrow} 0$, and $H$ is compact from $\mathscr{L}^{p_{t}}\left(\Omega_{1}\right)$ to $\mathscr{L}^{q_{t}}\left(\Omega_{2}\right)$.

Q.E.D.

Remark II.8. In [4] Krasnosel'skii proved Theorem 1.2 in the particular case when $\Omega_{2}$ is bounded, but he did not give a proof in the case when $\Omega_{2}$ is unbounded. I learned about [4] after this paper was completed.

Let us compare our result with the one obtained by the method of quadratic forms [2] (and the references to original contributions quoted there), [5], [6], [7], and [8].

For simplicity we consider the case $P_{0}+q(x)$. We define

$$
h_{0}(\phi, \psi)=\left(P_{0} \phi, \psi\right), \phi, \psi \in H_{m / 2,2} \text {. }
$$

$h_{0}$ is a closed bounded below ${ }^{3}$ quadratic form with $D\left(h_{0}\right)=H_{m / 2,2} \otimes H_{m / 2,2}$. The perturbation is the following form

$$
\begin{aligned}
& v(\phi, \psi)=(q \phi, \psi) \\
& D(v)=\left\{\phi, \psi \in \mathscr{L}^{2} \otimes \mathscr{L}^{2}|| v(\phi, \psi) \mid<\infty\right\} .
\end{aligned}
$$

The perturbed form is defined as follows

$$
\begin{aligned}
& h(\phi, \psi)=h_{0}(\phi, \psi)+v(\phi, \psi) \\
& D(h)=D\left(h_{0}\right) \cap D(v) .
\end{aligned}
$$

If $h$ extends to a closed bounded below form, $\tilde{h}$, then $\tilde{h}$ has an associated selfadjoint bounded below operator, $H$, which is an extension of $P_{0}+q . H$ is called the forms extension or the forms sum of $P_{0}$ and $q$.

Theorem II.9. Suppose that $q \in R_{m}$ and $\lim _{\boldsymbol{R} \rightarrow \infty} \int_{|y| \geqq R}|q(y)| G_{m}(y-x) d y=0$ uniformly in $x$.

Then $h=h_{0}+q$ is closed and bounded below with domain $H_{m / 2,2} \otimes H_{m / 2,2}$. Let $H$ be the associated selfadjoint, bounded below, operator. Then $H$ coincides with the operator $\mathrm{H}_{2}$ given by Theorem I.1, i.e. $\mathrm{H}_{2}=\mathrm{H}$.

Proof. We prove as in Lemma III.2 of [1] that $|q|^{1 / 2}$ is a compact operator from

3 Since $P_{0}$ is elliptic we can always reduce the problem to the case when $P_{0}$ is bounded below by a change in sign 
$H_{m / 2,2}$ to $\mathscr{L}^{2}$. Then for any $\varepsilon>0$ there exists $K_{\varepsilon}$ such that

$|v(\phi, \phi)| \leqq \varepsilon h_{0}(\phi, \phi)+K_{\varepsilon}(\phi, \phi) \phi \in H_{m / 2,2}$.

Then $h$ is closed in $D(h)=H_{m / 2,2} \otimes H_{m / 2,2}$, and bounded below. We prove below that for $b$ large enough $R_{2}(-b)=R_{0}^{1 / 2}(1-Q(-b))^{-1} R_{0}^{1 / 2}$, where $Q(z)=$ $R_{0}^{1 / 2}(z) q R_{0}^{1 / 2}(z)$. From this and the expression $(H \phi, \psi)=h(\phi, \psi), \psi, \phi \in D(H)$ it follows from a straight forward computation that we omit that $R_{2}(-b)=(-b-$ $H)^{-1}$. Then $H=H_{2}$. Let us prove now that $R_{2}=R_{0}^{1 / 2}(1-Q)^{-1} R_{0}^{1 / 2}$. Take $b$ large enough such that $b \in \rho\left(H_{2}\right)$ and: $\left\||q|^{1 / 2} R_{0}^{1 / 2}(-b)\right\|<1$. Then

$$
\begin{aligned}
& R_{2}(-b)=R_{0}^{1 / 2}(-b)\left(1-\left(|q|^{1 / 2} R_{0}(-b)^{1 / 2}\right)^{*}(\operatorname{sign} q)|q|^{1 / 2} R_{0}(-b)^{1 / 2}\right)^{-1} R_{0}^{1 / 2} \\
& =R_{0}^{1 / 2}(1-Q)^{-1} R_{0}^{1 / 2} .
\end{aligned}
$$

Finally, let us prove that the spectrum of $H_{p}$ is real for $1 \leqq p \leqq \infty$.

Theorem II.10. Suppose that $q \in R_{m}$ and $\lim \int_{R \rightarrow \infty}|q(y)| G_{m}(y-x) d y=0$ uniformly in $x$. Then the spectrum of $H_{p}, 1 \leqq p \leqq \infty$ is real.

Proof. Since $H_{p}^{*}=H_{p^{\prime}}, \frac{1}{p}+\frac{1}{p^{\prime}}=1, p<\infty$ it is enough to prove the Theorem for $2<p<\infty$.

The only spectrum that $H_{p}$ can have in $\mathbb{C} \backslash R$ is discrete spectrum. First assume that $q(x)$ is a bounded function. Define

$$
q_{R}(x)=\left\{\begin{array}{l}
q(x)|x| \geqq R \\
0 \text { otherwise. }
\end{array}\right.
$$

Let us define $H_{p, R}=P_{0, p}+q-q_{R}$.

Then $\lambda \in \mathbb{C} \backslash R$ is an eigenvalue of $H_{p, R}$ with eigenvector $\phi$ if and only if

$$
\phi=R_{0}(\lambda)\left(q-q_{R}\right) \phi .
$$

But since $\phi \in \mathscr{L}^{p}, p>2,\left(q-q_{R}\right) \phi \in \mathscr{L}^{2}$. Then $\phi \in \mathscr{L}^{2}$, and since the operator $H_{2, R}$ is selfadjoint in $\mathscr{L}^{2}$ we must have $\phi=0$. Then $H_{p, R}$ has no spectrum in $\mathbb{C} \backslash R$. But as operator in $\mathscr{L}^{1}$

$$
\left\|\left(q-q_{R}\right) R_{0}-q R_{0}\right\|_{\mathscr{L}^{1}, \mathscr{L}^{1}} \leqq K \sup _{x} \int_{|y| \geqq R}|q(y)| G_{m}(x-y) d y \underset{R \rightarrow \infty}{\longrightarrow} 0
$$

by assumption.

Then $H_{1, R}$ converges in resolvent sense to $H_{1}$. By taking adjoint $H_{\infty, R}$ converges in resolvent sense to $H_{\infty}$ and by interpolation $H_{p, R}$ converges to $H_{p}$ in resolvent sense. Since $H_{p, R}$ has no non real eigenvalues $H_{p}^{p, R}$ has no non real eigenvalues, and then it has no non real spectrum at all. If $q$ is not bounded define

$$
q_{l}(x)=\left\{\begin{array}{l}
q(x) \text { if }|q(x)| \leqq l \\
0 \text { otherwise }
\end{array}\right.
$$

Then $H_{p, l}=P_{0, p}+q_{l}$ has no non real eigenvalues. But, $\left\|q R_{0}-q_{l} R_{0}\right\|_{\mathscr{L}^{1}, \mathscr{L}^{1}} \leqq$ $K R_{m}\left(q-q_{l}\right)$. As in Lemma III.2 of [1] we prove that $R_{m}\left(q-q_{l}\right) \underset{l \rightarrow \infty}{\longrightarrow} 0$. Then 
$H_{l, 1}$ converges to $H_{1}$ in resolvent sense, and as before we prove that $H_{l, p}$ converges to $H_{p}$ in resolvent sense. $\quad$ Q.E.D. We considered the case $P_{0}+q$ in Theorems II.9 and II.10 by simplicity, but these theorems extent to the general case $\mathrm{P}_{0}+\sum_{i=1}^{M} q_{i} Q_{i}$.

Acknowledgements. I thank Professor Martin Schechter for very useful conversations.

\section{References}

1. Weder, R. : Second order operators in the uniform norm. Preprint Harvard University (1977)

2. Kato, T. : Perturbation theory for linear operators. Berlin-Heidelberg-New York: Springer 1966

3. Schechter, M. : Spectra of partial differential operators. Amsterdam: North Holland 1971

4. Krasnosel'skii,, M. A. : On a theorem of M. Riesz. Soviet Math. 1, 229-231 (1960)

5. Schechter, M.: On the invariance of the essential spectrum of an arbitrary operator II. Ric. Mat. 16, 3-26 (1967)

6. Faris, W. G. : The product formula for semigroups defined by Friedrichs extensions. Pacific J. Math. 22, 47-70 (1967)

7. Nelson, E. : Topics in dynamics. Princeton: Princeton University Press 1969

8. Simon, B. : Halmiltonians defined as quadratic forms. Commun. math. Phys. 21, 192-210 (1971)

9. Koller, H., Schechter, M., Weder, R. : Ann. Inst. Henri Poincaré 26, 303-311 (1977)

10. Weder, R. : The unified approach to spectral analysis II. Preprint Princeton University (1977)

Communicated by H. Araki

Received September 20, 1977 
